\title{
Development and evaluation of a novel flex sensor- based glenohumeral subluxation degree assessment for wearable shoulder sling
}

\section{Shuangyuan Huang}

South China University of Technology https://orcid.org/0000-0002-4258-8311

Jiaming Zhou

Maoming People's Hospital

\section{Yude Yang}

Maoming People's Hospital

\section{Zhonghua Chen}

Maoming People's Hospital

\section{Shiman $\mathrm{He}$}

South China University of Technology

\section{Lilin Chen}

Third Affiliated Hospital of Sun Yat-Sen University Department of Rehabilitation Medicine

\section{Haiqing Zheng}

Third Affiliated Hospital of Sun Yat-Sen University Department of Rehabilitation Medicine

Zhenhong Liang

Maoming People's Hospital

Longhan Xie ( $\square$ melhxie@scut.edu.cn )

South China University of Technology https://orcid.org/0000-0002-5137-1413

\section{Research}

Keywords: Flex sensor, Glenohumeral subluxation assessment, Stroke rehabilitation, Wearable shoulder sling

Posted Date: April 2nd, 2021

DOI: https://doi.org/10.21203/rs.3.rs-340654/v1

License: (c) (1) This work is licensed under a Creative Commons Attribution 4.0 International License.

Read Full License 
Version of Record: A version of this preprint was published at Sensors and Actuators A: Physical on February 1st, 2022. See the published version at https://doi.org/10.1016/j.sna.2022.113405. 


\title{
RESEARCH
}

\section{Development and evaluation of a novel flex sensor-based glenohumeral subluxation degree assessment for wearable shoulder sling}

\author{
Shuangyuan Huang ${ }^{1 \dagger}$, Jiaming Zhou ${ }^{2 \dagger}$, Yude Yang ${ }^{2}$, Zhonghua Chen ${ }^{2}$, Shiman He ${ }^{1}$, Lilin Chen ${ }^{3}$, Haiqing
} Zheng $^{3}$, Zhenhong Liang ${ }^{2 *}$ and Longhan $\mathrm{Xie}^{1^{*}}$

Sample of title note

\footnotetext{
${ }^{*}$ Correspondence:

1454825714@qq.com;

melhxie@scut.edu.cn

${ }^{2}$ Department of Rehabilitation

Medicine, Maoming people's

Hospital, 525000 Maoming, China

${ }^{1}$ Shien-Ming Wu School of

Intelligent Engineering, South

China University of Technology,

510640 Guangzhou, China

Full list of author information is

available at the end of the article

†Shuangyuan Huang and Jiaming

Zhou contributed equally to this

work as first authors
}

\begin{abstract}
Background: Glenohumeral subluxation (GHS) is reported in as many as $81 \%$ of patients with stroke, which presents considerable challenges for individuals involved in stroke rehabilitation. During rehabilitation, the joint head of the glenohumeral joint was maintained in the socket by wearing a GHS shoulder sling. To ensure that the glenohumeral joint head is in the socket, an orthosis is required to automatically adjust the degree of orthosis according to the degree of GHS. However, there is currently no wearable method for assessing the degree of GHS. This study aims to develop a GHS assessment method for patients with stroke that has the potential to be integrated into a wearable shoulder sling.
\end{abstract}

Methods: To that end, we developed a GHS assessment method for patients with stroke based on a flex sensor, which has the potential to be integrated into a wearable shoulder sling. Seventeen patients with stroke in a diverse degree of GHS were recruited for the study. The curvature difference of the affected shoulder in the subluxation and reduction states was collected by flex sensor to calculate the degree of GHS (AGTD). Meanwhile, the GHS degree, calculated as the acromion-greater tuberosity distance di.fference (AGTD) of the affected and unaffected shoulder, was measured by the gold-standard radiographic assessment.

Results: The GHS degree measurement by flex sensor and radiographic assessment has strongly correlated $(r=0.92, p<0.01)$. The relationship of GHS degree measured by radiographic assessment (AGTD) and flex sensor (AGHS) is linear according to an interpolating curve with an equation: $A G H S=0.767 A G T D+$ $1.781\left(R^{2}=0.84, R M S E=1.61\right)$. The method has excellent reliability with $R=0.87, C I=95 \%$ for assessment of GHS degrees.

Conclusions: The proposed method demonstrates good performance, is wearable, easy to use, and allows automatic measurements, so as to be a valuable method to assessment of GHS.

Keywords: Flex sensor; Glenohumeral subluxation assessment; Stroke rehabilitation; Wearable shoulder sling

\section{Introduction}

Glenohumeral subluxation (GHS) is reported to be present commonly in $17-81 \%$ of individuals with paralyzed shoulder muscles following stroke [1,2], which is defined as "increased translation of the humeral head relative to the glenoid fossa" [3]. 
Compared to a healthy shoulder, the GHS shoulder shows a palpable gap and an obvious change in the contour curve of the shoulder joint between the acromion and the humeral head [4]. GHS presents considerable challenges to the rehabilitation of the upper limb, such as impaired normal shoulder function, and prolonged hospital stays other post-stroke complications such as hemiplegic shoulder pain and poor motor recovery [5].

To reduce GHS post-stroke, the shoulder slings are most frequently used among the various shoulder sling [6-8]. In the clinic, the use of shoulder slings aims to maintain the mechanical integrity of the glenohumeral joint that keeping the humeral head in the glenoid fossa, which allows the glenohumeral joint to produce rotation [6]. When donned the slings, the humeral head would be manually adjusted to the glenoid fossa based on the GHS degree to regain the shoulder mechanical integrity [9]. However, because of the gravity and activity of the upper limb, the glenohumeral joint head would easily slides out of the glenoid fossa. None of these shoulder slings could be able to monitor and automatically keep the humeral head within the glenoid fossa, which is an important factor that the GHS sling treatment is inconclusive in previous studies [10]. A main challenge of the GHS sling to implement this function is the lack of wearable and objective GHS measurement methods [11].

Current GHS clinical measurement includes radiographs [12], ultrasound [4], fingerbreadth palpation [13], and other auxiliary instruments, such as calipers [14] and thermoplastic jig [15]. However, these methods are difficult to be integrated into the wearable GHS sling. Besides, they have other defects to monitor the GHS degree and whether the humeral head in the glenoid fossa, such as radiation exposure of radiographs [16], professional imaging knowledge ,and skilled operation skills requirements of ultrasound $[17,18]$ and subjective of finger palpation [4].

The flex sensors, which can be shaped to fit in body joints [19], are produced by filming a special carbon ink printed on a polyester support material and changing its total resistance when bent. They have been widely used as electronic goniometers in wearable rehabilitation devices, such as to measure trunk postures garments [20], rehabilitation cybernetic glove [21], and knee flexion angle measurement system [22], to assess the movement of body segments [19,23-25].

According to the need for wearable GHS sling, and inspired by the curvature change in GHS shoulder joint appearance, we tried to assess the GHS degree to monitor the mechanical integrity of the glenohumeral joint by using a flex sensor.

The purpose of this article is to develop and evaluate a novel GHS degree assessment method based on a flex sensor for the wearable shoulder sling. This study hypothesized that the curvature difference of affected shoulder between subluxation and reduction status could reflect the degree of GHS, the difference of shoulder contour curve could be obtained by flex sensor. There were 19 patients with a diverse degree of GHS who participated in the study. The GHS degree was assessed by the gold-standard radiographic assessment and clinical-used fingerbreadth palpation. The GHS degree, calculated as the acromion-greater tuberosity distance difference (AGTD) of the affected and unaffected shoulder, was measured by radiographic assessment. Curvature difference collected by flex sensor (AGHS) has strong correlated $(r=0.92, p<0.01)$ with AGTD. The relationship of GHS degree 
measured by radiographic assessment (AGTD) and flex sensor (AGHS) is linear according to an interpolating curve with the equation: $A G H S=0.767 A G T D+1.781$ $\left(R^{2}=0.84, R M S E=1.61\right)$. The intra-class correlation coefficient (ICC) of repeated measurements was $R=0.87, C I=95 \%$, which indicated that this method had good reliability. This method has the potential to be integrated with a GHS sling to improve stroke rehabilitation outcomes.

\section{Methods}

Participants

Seventeen patients ( 11 men and 6 women, mean age 52 years, mean weight 58 $\mathrm{kg}$ ) with post-stroke hemiplegia and different degrees of GHS were included in this study, as shown in Table 1. We excluded patients with previous shoulder surgery, aphasia, or cognitive impairment which could interfere with their understanding of instructions during assessment, and severe motor impairment who could not maintain an independent upright sitting position when taking the radiograph.

The selection of patients with stroke was performed with the supervision of at least two professional rehabilitation therapists. Ethics approval and consent to participate (i.e., Informed consent) were obtained from all participants to complete the protocol approved by the Maoming People's Hospital Department of Ethics Committee. The study was carried out under the Declaration of Helsinki.

Table 1 Details of the seventeen participants with stroke

\begin{tabular}{ccccccc}
\hline & Sex & Age (year) & Heitht $(\mathrm{cm})$ & Weight $(\mathrm{kg})$ & L/R & AGTD $(\mathrm{mm})$ \\
\hline Details & $11 \mathrm{M}, 6 \mathrm{~F}$ & $52 \pm 16$ & $168 \pm 10$ & $58 \pm 12$ & $14 \mathrm{~L}, 3 \mathrm{R}$ & $7.25 \pm 5.04$ \\
\hline
\end{tabular}

\section{Radiographic assessment}

Radiographic assessment (RA) is considered a standard measurement for evaluating GHS degree, has been used in several studies to assess the effectiveness of therapy or development of GHS over time [12]. Generally, the RA approach is reliable and valid. Patients were seated and arms dependent in a neutral position. The GHS degree, calculated as the acromion-greater tuberosity (AH) distance difference (AGTD) of the affected and unaffected shoulder [17], shown in Fig. 1. Two specialists in imaging performed the radiographic measurements independently. The AGTD was calculated as follows:

$$
A G T D=A H_{a}-A H_{u}
$$

where $A H_{a}$ is the affected shoulder AH distance and $A H_{u}$ is the unaffected shoulder AH distance. The AGTD was $7.25 \pm 5.04 \mathrm{~mm}$ for the participants.

\section{Fingerbreadth palpation}

Measurement of GHS is mainly carried out by fingerbreadth palpation (FP) in the clinic. The number of fingerbreadths that can be inserted between the inferior aspect of the acromion and the superior aspect of the humeral head [1]. A standardized protocol was used [4] as shown in Fig. 2 (a). Participants were seated in a chair with both feet flat on the ground. The physical therapist first assessed the unaffected 


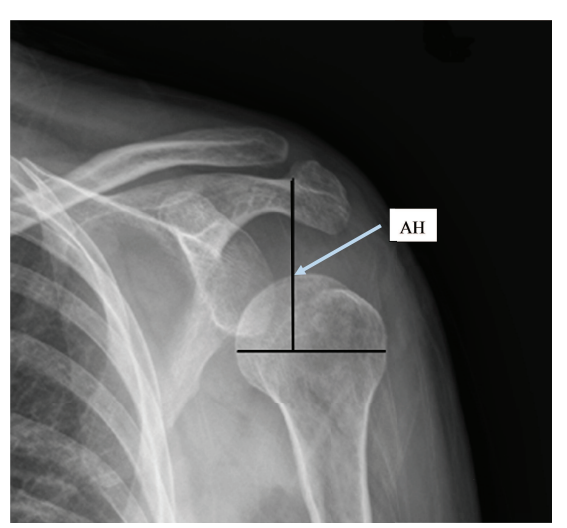

Figure 1 Measurement of GHS degree from radiograph, which measured from the acromial point to the central point of the humeral head.

side to palpate the gap between the acromion and the head of the humerus, and this assessment was repeated on the affected shoulder. Shoulders were positioned in neutral rotation, with the arm hanging by the side (thumb pointing forward) close to the body with no abduction. The GHS degree assessed by FP was defined by a 0-3 grading scheme: 0 no subluxation, $1=0-1$ fingerbreadth gap $(\mathrm{FG}), 2=1-2$ fingerbreadth gap, $3=>2$ fingerbreadth gap [13]. The FP results will be discussed in the Discussion section.

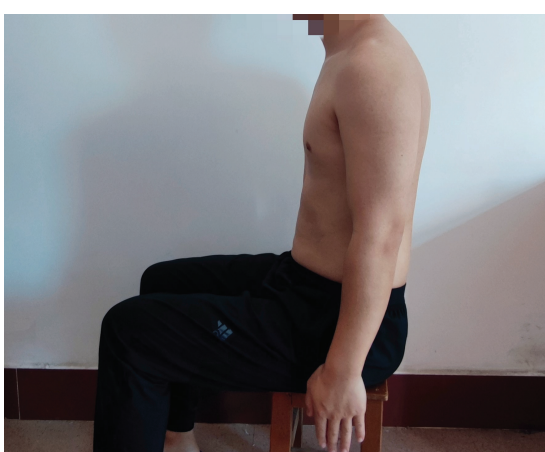

(a)

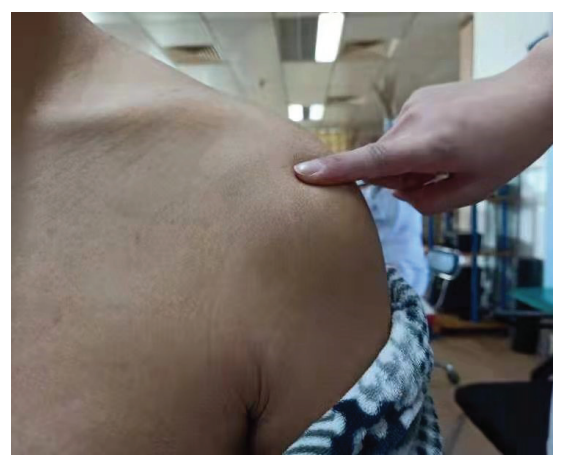

(b)

Figure 2 Standardized position for fingerbreadth palpation data collection (a) and fingerbreadth palpation (b).

\section{Materials and Data Acquisition}

\section{Flex sensor}

The flex sensor is composed of a single, thin and flexible plastic substrate and a resistive layer of proprietary carbon ink, which is screen printed on the flex sensor to make it suitable for a particular body joint. When the layer is bent, the ink separates into many micro-cracks, which upon movement open/close according to the specific bending angle of the material, determining a decrease/increase of the material conductivity $[26,27]$, shown in Fig. 3. This type of sensor is available on the market (Flexpoint Sensor Systems Inc). According to the required length of the 
shoulder joint, we chose a flex sensor with a length of 4 inches. With a typical flex sensor, a flex of 0 degrees gives $5 k-10 k$ resistance, and a flex of 90 gives $10-20$ $\mathrm{K} \Omega$.

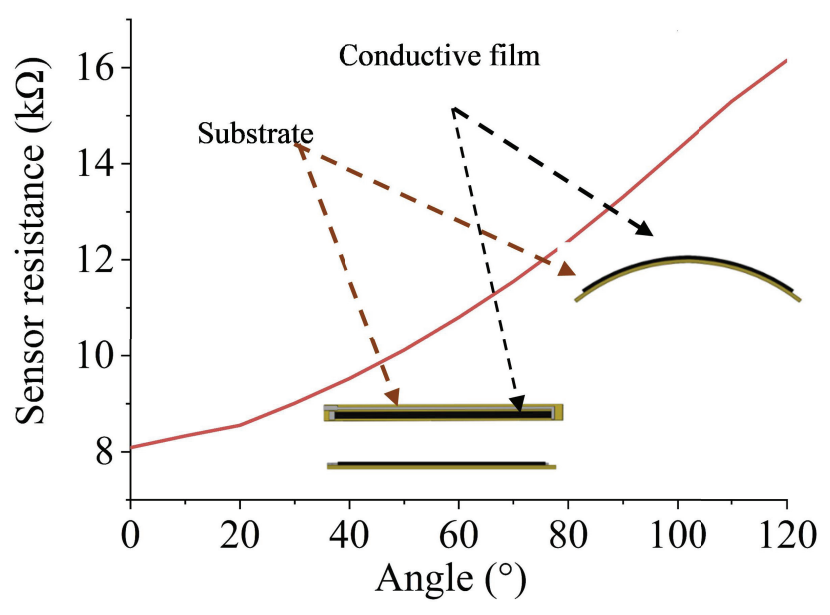

Figure 3 Scheme of the flex sensor.

Flex sensor calibration Due to the influence of manufacturing factors such as sheet resistance, active layer thickness, shape, and size, resistance performance among flex sensor samples would be inconsistent. Before starting the experimental analysis, the flex sensor should be calibrated [26]. The schematic of the experimental set up showed in Fig. 4 (a). The selected sensor samples were laid as a cantilever beam on a hinge. The sensor side connected to the electrodes was locked in a stationary clamp, fixed to a rotating platform operated by a step motor. The other side of the sensor is free of slipping in a sliding clamp, to avoid any stretching effect to the sample, which must be characterized only against the bending angle.

The basic circuit diagram of a flex sensor, shown in Fig. 4 (b) [28], the voltage output of the flex sensor that can be determined as

$$
R_{1}=\frac{V_{\text {in }} R_{2}}{V_{\text {in }}-V_{\text {out }}}
$$

Where $R_{1}$ is the resistance of flex sensor, $R_{2}$ is the resistance of resistor, $V_{i n}$ is power supplied to circuit, $V_{\text {out }}$ the voltage.

The nonlinear region of the resistance-angle regression of the flex sensor is mainly concentrated in the region less than $50^{\circ}$ [31]. The curvature of the shoulder tends to be greater than $60^{\circ}$, so we only calibrated the linear areas, greater than $60^{\circ}$. The resistance behavior of flex sensors was measured against outward bending angles from $60^{\circ}$ to $120^{\circ}$, with the step of $10^{\circ}$ and a hold-time of $2 \mathrm{~s}$ between them. This protocol was repeated five times to test repeatability.

The relationships between resistance against flexion angles of the flex sensor during the calibration test, shown in Fig. 5. The flex sensor resistance has linear relationships against bending angle. First-order polynomials can well fit these linear 


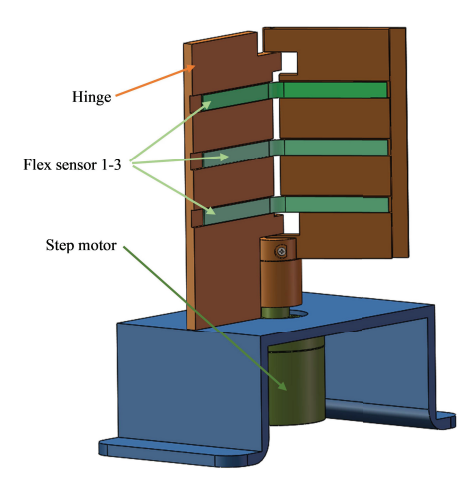

(a)

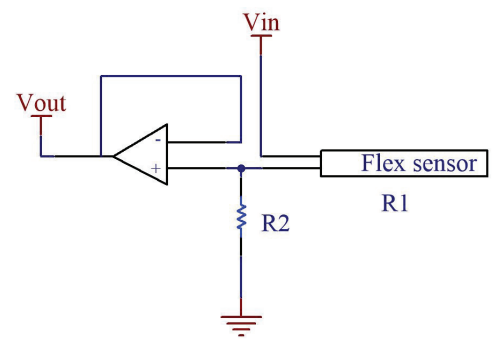

(b)

Figure 4 Experimental set-up used to characterize the sensor device against the bending angle (a) and basic circuit diagram of a flex sensor (b).

relationships $\left(R^{2} \geq 0.9997\right)$. Because the resistance-angle regression performance between the sensors is similar, we randomly selected the sample of sensor 3 as the detection sensor in this study. Since the resistance-angle regression is linear, in the actual application process, the flex sensor only needs to measure two sets of resistance-angle values to obtain the resistance-angle regression relationship.

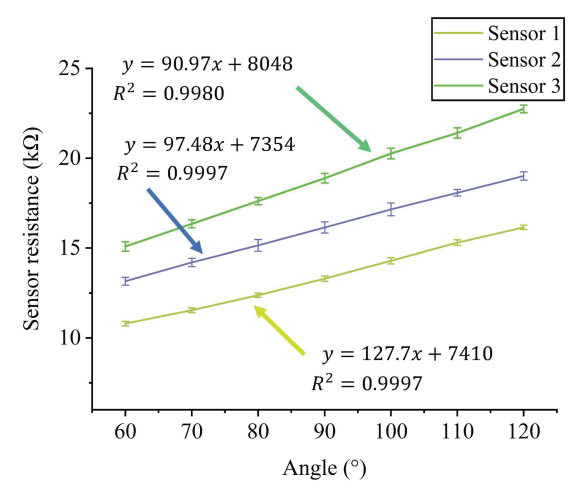

Figure 5 Static characterization and relationship of sensor resistance against outward bending angles, as total mean and standard deviation either inside measurements on the same sample and between 5 samples of each sensor. 
The Intraclass Correlation Coefficient (ICC) of the repeated calibration measurement of the selected flex sensor samples $(R=0.999 \pm 0.0006, C I=95 \%)$, indicated excellent reliability for flex sensor sensing the angle changes.

GHS band The wearable GHS sensory band (GSB) has the sensor embedded in a track, directly located on the shoulder, shown in Fig. 6. An intramuscular tape on the outer layer of the track to ensure that the sensor was firmly attached to the skin. The one edge of the flex sensor is fastened on shoulder peak by the shoulder band of GSB. The other edge of the sensor slips freely in the track to avoid any stretching effect which increased the resistance value. The GHS allow an easy adjustment of the GSB to the anatomy of the participant's shoulder.

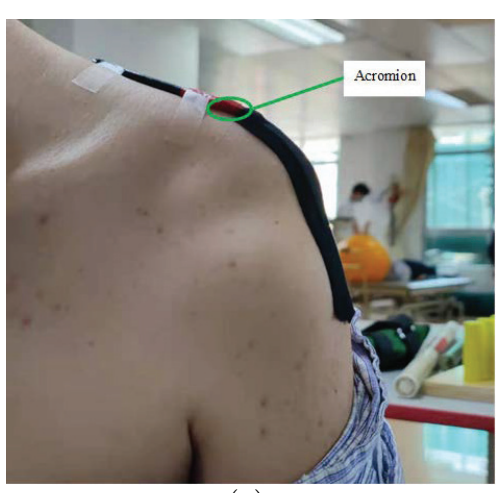

(a)

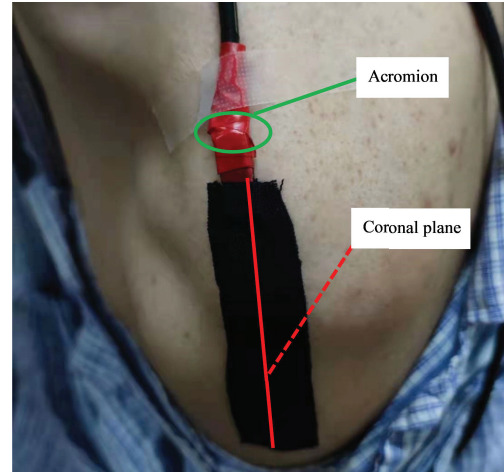

(b)

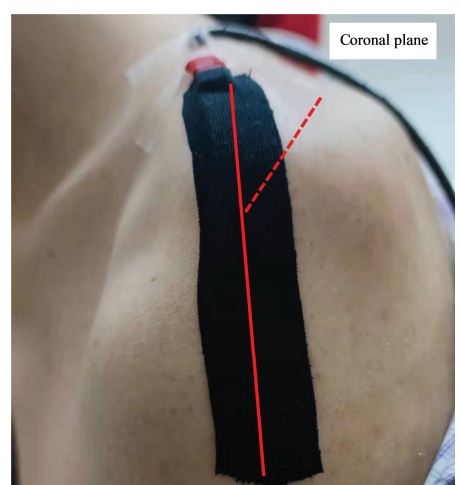

(c)

Figure 6 Front (a), left (b), and upper (c) views of GSB placed on the shoulder joint.

Flex sensor data acquisition

According to the objective of this article, the GHS degree was measured the affected shoulder curvature difference between subluxation and reduction status. The GSB, starting at the acromion, is located along the coronal plane, shown in Fig. 6. The flex sensor data were recorded at a measurement frequency of $50 \mathrm{~Hz}$.

During data collection, shoulders were positioned in neutral rotation, with the arm hanging by the side (thumb pointing forward) close to the body with no abduction [4]. The therapist placed one hand on the acromion to prevent compensatory movements in the other joints of the shoulder. The other hand gave the patient arm 
gravity compensation to restore the shoulder joint and then held it for about 10 seconds, shown in Fig. 7 . The reduced shoulder joint is shown in Fig. 8 (b). The therapist then removed the compensating force so that the shoulder was subluxated again and then held it for about 10 seconds. The subluxated shoulder is shown in Fig. 8 (a). Five values of the flex sensor resistance difference of subluxation and reduction were measured.

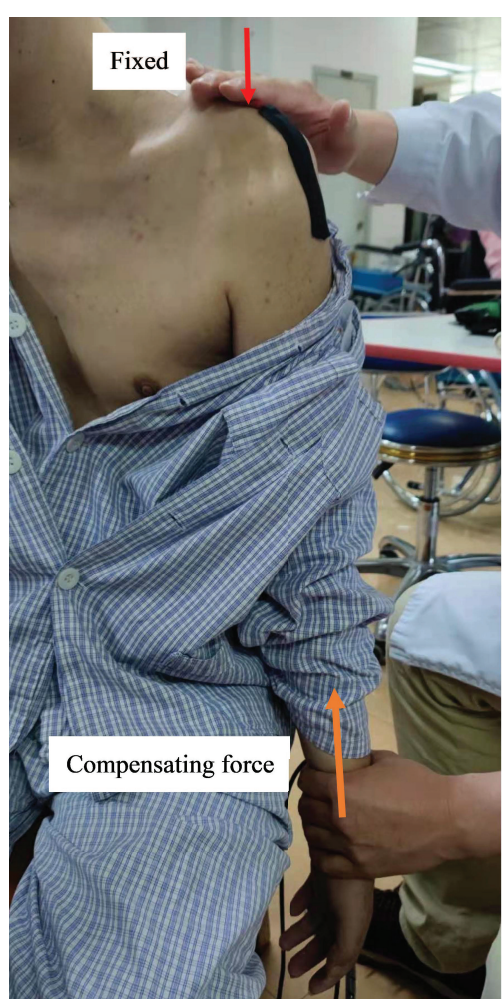

Figure 7 Therapist's action of reducing the patient's shoulder joint.

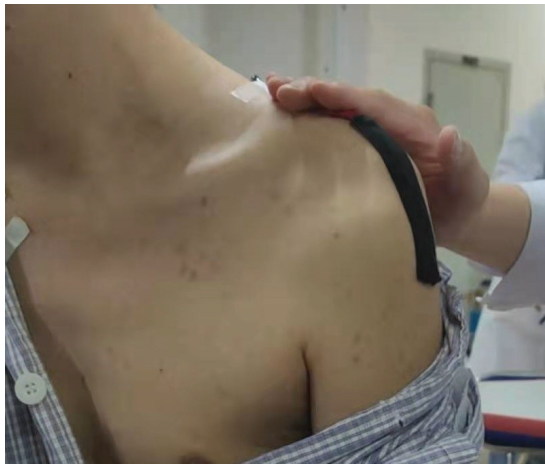

(a)

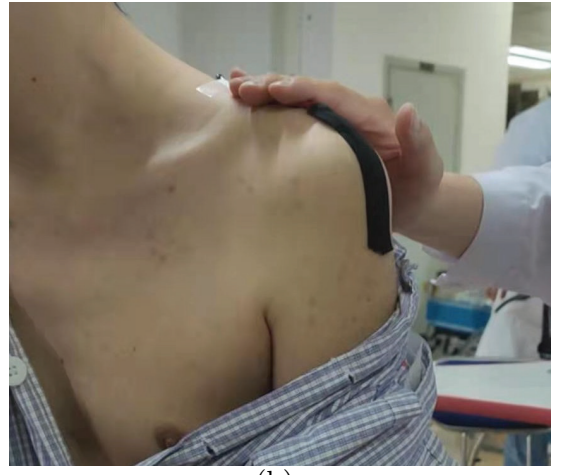

(b)

Figure 8 (a) The shape of shoulder the joint in subluxation. (b) The shape of the shoulder joint in reduction. 


\section{Data processing}

Data segment Segmentationn

The raw data were first smoothed by a moving average filter window.

$$
S S(i)=\left\{\begin{array}{l}
\frac{1}{2 i-1} \sum_{1}^{2 i-1} S(i), i<\frac{l}{2} \\
\frac{1}{w} \sum_{i-\frac{l}{2}}^{i+\frac{l}{2}} S(i), \frac{l}{2}<i<g-\frac{l}{2} \\
\frac{1}{2 i-1} \sum_{g-(2 i-1)}^{g} S(i), i>g-\frac{l}{2}
\end{array}\right.
$$

Where $S(i)$ is the raw data of the flex sensor.w $=50$ is the window length. $g$ is the length of raw data. By comparing the changes before and after reduction to represent the degree of subluxation, we need to distinguish the values of dislocation state and reduction state. In the process of providing gravity compensation, the curvature of the shape of the shoulder joint will increase, and the resistance value will have a rise, so we regard the increase of the resistance value before and after as the subluxation $\left(K_{s b}\right)$ and reduction $\left(K_{r e}\right)$ state.

$$
\begin{aligned}
K_{s} b & =\frac{\sum_{i=t_{1}-n}^{t_{1}} S S(i)}{n} \\
K_{r} e & =\frac{\sum_{i=t_{2}}^{t_{2}+n} S S(i)}{n}
\end{aligned}
$$

Where $n=50$, and $t_{1}=\arg \min _{i} S S^{\prime \prime}(i), t_{2}=\arg \max _{i} S S^{\prime \prime}(i)$. An example of data segmentation is shown in Fig. 9.

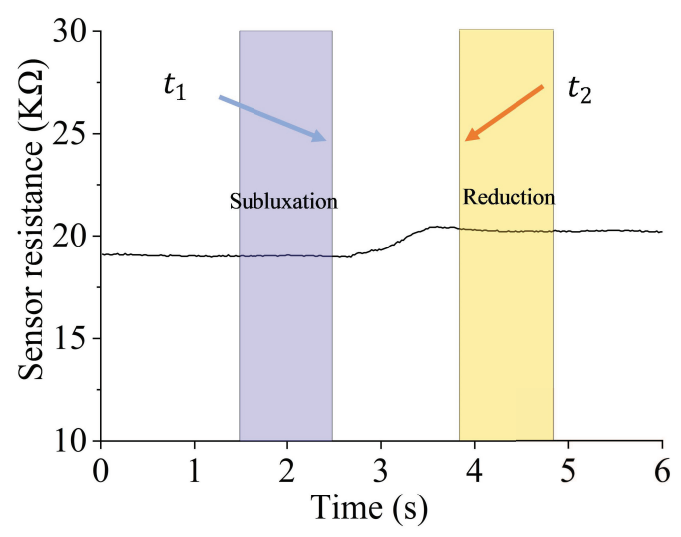

Figure 9 Example of data segmentation.

The difference between the curvature of shoulder subluxation and reduction statue was selected to represents the GHS degree. The GHS degree detected by the flex sensor AGHS were computed:

$$
\begin{aligned}
& A G H S_{z j}=L^{-1}\left(K_{r e_{z j}}\right)-L^{-1}\left(K_{s b_{z j}}\right) \\
& A_{G H S_{z}}=\frac{\sum_{j=1}^{N} A G H S_{z j}}{N}
\end{aligned}
$$


where $L(K)$ is the resistance-angle regression relationship, shown in Fig. $5 . z j$ being the $j^{t} h$ assessment of $z^{t} h$ participant. $N=5$ is the number of repetitions.

\section{Statistical analysis}

Statistical analysis was performed by IBM SPSS Statistics v. 24.0 for Windows (Armonk, NY: IBM Corp.). Pearson's rank correlation coefficients $(r)$ analysis was used to evaluate test relationships between different measurement methods. The $\mathrm{r}$ has the result has a value between -1 and 1 . The greater the absolute value of $r$, the larger the strength of association based on the following categories:0.1-0.3 (small), $0.3-0.5$ (medium), $0.5-1$ (large). The Intraclass Correlation Coefficient (ICC) was used to assess intra-rater reliability of repeated flex sensor measurements. The meaning of ICC value $(R)$ is shown in Table 2 [32].

Table 2 The interpretation of ICC value.

\begin{tabular}{cc}
\hline$R$ & Interpretation \\
\hline $0.00<|R|<0.40$ & Poor \\
$0.40<|R|<0.59$ & Fair \\
$0.60<|R|<0.74$ & Goof \\
$0.75<|R|<1.00$ & Excellent \\
\hline
\end{tabular}

\section{Results}

In this study, the mean and range of the curvature difference of the affected shoulder between subluxation and reduction status measured by flex sensor are $7.22 \pm 3.84$ and 13.57 respectively. Flex sensor GHS assessment is a strong correlation $(r=0.92, p<$ $0.01)$ with RA. The results indicate that the curvature difference of the affected shoulder between subluxation and reduction status can assess the degree of GHS and can be measured by the flex sensor. The relationship of GHS degree measured by RA (AGTD) and flex sensor (AGHS) is linear according to an interpolating curve with the equation: $A G T S=0.767 A G T D+1.781\left(R^{2}=0.84, R M S E=1.61\right)$, shown in Fig. 10. The result is due to the increase of GHS degree and the increase of glenohumeral gap, the curvature of the affected shoulder will change from convex to flat or even slightly concave.

The ICC of the repeated GHS measurement of the selected flex sensor samples $(R=0.87, C I=95 \%)$, indicated excellent reliability for flex sensor assessment of GHS degrees.

\section{Discussion}

To the best of our knowledge, this study is to develop and evaluate a novel GHS degree assessment method based on the flex sensor for the wearable shoulder sling for the first time. The degree of GHS, expressed by curvature difference of affected shoulder between subluxation and reduction status, collected by flex sensor has a strong correlation $(r=0.92, p<0.01)$ with RA. ICC of repeated measurements is $R=0.87, C I=95 \%$, which indicated that this method had good reliability. The relationship of GHS degree measured by RA (AGTD) and flex sensor (AGHS) is linear in terms of an interpolating curve with equation: $A G H S=0.767 A G T D+$ $1.781\left(R^{2}=0.84, R M S E=1.61\right)$. The obtained results confirm such methodology as a valuable approach to the measurement of GHS degree in a reliable, simple, and 


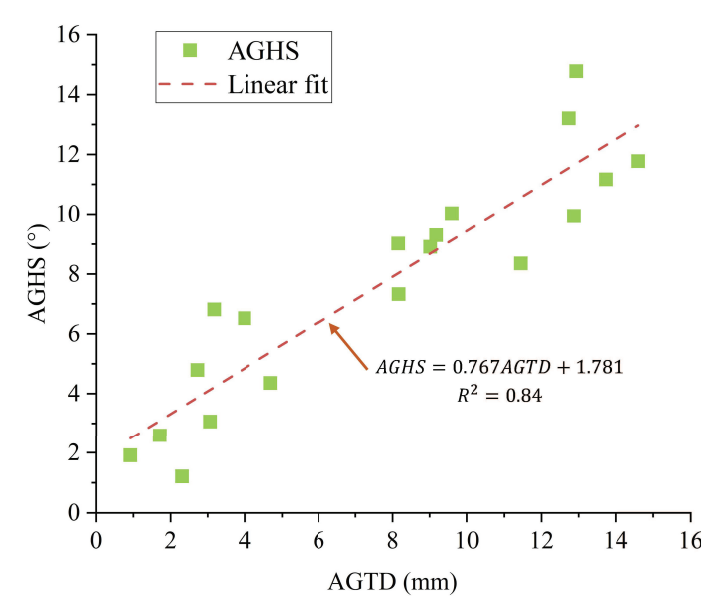

Figure 10 The relationship between the GHS degree measured by radiographic assessment (AGTD) and the GHS degree measured by Flex sensor (AGHS).

cheap way. The proposed method of detection GHS has the potential to perform with GHS sling and improve rehabilitation efficacy.

The reported correlations for the concurrent validity of the fingerbreadth palpation method in comparison with RA range from 0.42 to 0.76 [13], and the similar result $(r=0.73, p<0.01)$ in our study, which is described as relatively low [29]. The lower correlations between radiographs and fingerbreadth palpation may reflect a variety of problems related to clinical stability of subluxation and changes in the subjects over time [4]. Compared with fingerbreadth palpation, the most commonly used clinical diagnosis method, the flex sensor method has higher correlations $(r=0.92, p<0.01)$ in comparison with radiographic measurements. The fingerbreadth palpation method has the advantage of being a quick, equipment-free method. However, because it is subjective, the ICC between raters is large range $(R=0.77,0.89, C I=95 \%)$ [15]. Ultrasound detection of GHS has the characteristics of non-invasive and high repeatability $(R=0.9580 .015, C I=95 \%)$. However, for therapists, this method, requiring specialized imaging skills and knowledge, is difficult to grasp. Ultrasound has incomparable advantages in evaluating shoulder pain caused by soft tissue injury of shoulder joint after stroke [30]. If the shoulder is tested only to detect the degree of subluxation, then the equipment and labor costs are not cost-effective.

In the preliminary experiment of this study, we also collected the curvature of the shoulder joint on the unaffected side and the affected side to explore whether the curvature difference on both sides could represent the degree of subluxation. Because the shape of the normal shoulder joint is symmetrical, the shape of the bilateral shoulder joint in patients with stroke and GHS is often asymmetric. However, the asymmetry caused by GHS cannot be detected by the flex sensor. Because of the reduction of muscle strength and inability to move, patients with GHS are often accompanied by muscle atrophy, which will have a great impact on the shape of the shoulder joint.

What's more, comparing the curvature of affected and unaffected side, the flex sensor needs to be held at a fixed angle. When held in a fixed bent position, the 
sensor resistance value would drift [31,32], shown in Fig. 11. Drift amount is mainly affected by manufacturer, coating, and sensor length. Because of time-varying creep behavior, the flex sensor not suitable for sensing the magnitude angle values, but it does not affect sensing the angle changes [31]. If the creep of the flex sensor can be overcome in the future, we can study assessing the degree of GHS by comparing the curvature of the affected and unaffected shoulder.

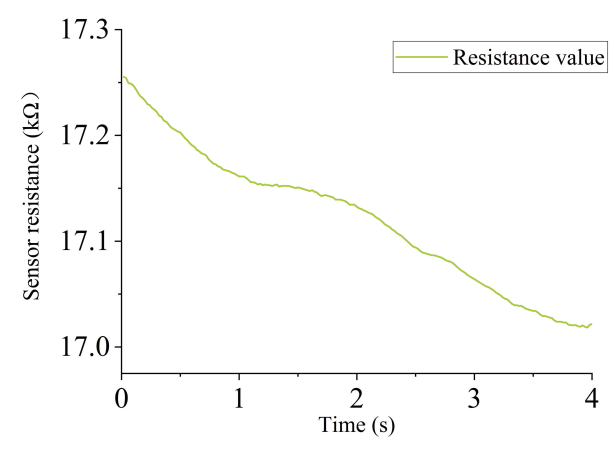

Figure 11 The drift of flex sensor resistance value with time.

Since we only use the linear phase of the sensor (about $50-120$ ), there is no need for tedious calibration process in the actual practice. All we need to calibrate is take the two calibration points and get the slope of the angle-resistance relationship.

Other studies can be performed in the future. Firstly, studying the feasibility and reliability of flex sensor for early GHS detection. If the flex sensor can detect the occurrence of GHS, therapists can intervene promptly, rather than wearing shoulder slings for all patients with low muscle strength. Because the abuse of a shoulder sling can cause secondary injuries such as impeding blood circulation to the affected limb and interfering with normal rehabilitation training. Secondly, in this paper, we focus on vertical GHS in stroke patients, and anterior GHS of the shoulder joint is also common after stroke. We will study whether flexible sensors can be used to evaluate the degree of anterior GHS for our next work. Thirdly, for the future development of wearable intelligent orthosis for GHS, a flex sensor can be used to monitor the orthosis status and prevent under-correction and over-correction.

\section{Conclusion}

In this paper, we developed a detection method for the degree of GHS in patients with stroke based on a flex sensor. Seventeen patients with stroke in different degrees of GHS were recruited to participate in the study to verify the feasibility and reliability of this method. This method yielded has strongly correlated $(r=0.92, p<0.01)$ with GHS degree measured by radiographic assessment. The relationship of GHS degree measured by radiographic assessment (AGTD) and flex sensor (AGHS) is linear according to an interpolating curve with the equation: $A G H S=0.767 A G T D+1.781\left(R^{2}=0.84, R M S E=1.61\right)$. The method has excellent reliability with $R=0.87, C I=95 \%$ for assessment of GHS degrees. Compared to the existing three methods, the proposed method is easy to perform with GHS 
orthoses and supporters for real-time adjustments of orthopedic parameters to improve orthosis and supporter effectiveness and safety.

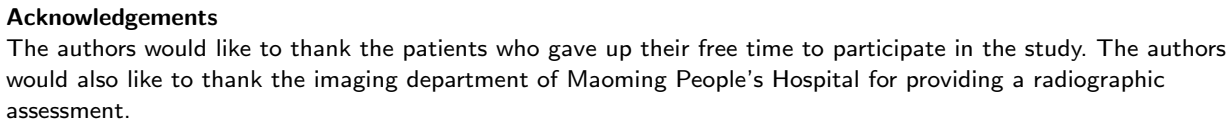

\section{Funding}

This work was supported in part by the National Natural Science Foundation of China (Grant No. 52075177), Joint Fund of the Ministry of Education for Equipment Pre-Research (Grant No. 6141A02033124), Research Foundation of Guangdong Province (Grant No. 2019A050505001 and 2018KZDXM002), Guangzhou Research Foundation (Grant No. 202002030324 and 201903010028), Zhongshan Research Foundation (Grant No.2020B2020), and Shenzhen Institute of Artificial Intelligence and Robotics for Society (Grant No. AC01202005011)...

Abbreviations

GHS: Glenohumeral subluxation;AGTD: Acromion-greater tuberosity distance difference; AGHS: Curvature difference collected by flex sensor; ICC: Intra-class correlation coefficient;RA: Radiographic assessment; $\mathrm{AH}$ :Acromion-greater tuberosity;FP: Fingerbreadth palpation; FG: Fingerbreadth gap; M: Mam; F: Female; L: Left; R: right; GSB: GHS sensory band...

Availability of data and materials

All data generated or analysed during this study are included in this published article and its supplementary information fles.

Ethics approval and consent to participate

This study was registered with and approved by the Medical Ethics Committee of Maoming people's Hospital, PJ2020MI-K179-01.

Competing interests

None to declare.

Consent for publication

Not applicable.

Authors' contributions

SH, LX, and HZ defned the study protocol. JZ,YY,ZC, and ZL were responsible for participants' recruitment. SH, JZ,YY and LC carried out the study and collected the patients' data. SH, LX performed the data analysis and wrote the manuscript. $\mathrm{SmH}$ participated in the study coordination and design. $\mathrm{SH}$ and $\mathrm{SmH}$ participated in the trials, providing the technology that was used during the evaluation, and gave technological support during the trials. They also provided information regarding new technology in the manuscript. All authors read and approved the fnal manuscript.

Authors' information

1Shien-Ming Wu School of Intelligent Engineering, South China University of Technology, Guangzhou, Guangdong Province, 510640, P. R. China. 2Department of Rehabilitation Medicine, Maoming people's Hospital, Maoming, 525000, China.3Department of Rehabilitation Medicine, The Third Affiliated Hospital, Sun Yat-sen Univerity, Guangzhou, 510630, China.

Author details

${ }^{1}$ Shien-Ming Wu School of Intelligent Engineering, South China University of Technology, 510640 Guangzhou, China. ${ }^{2}$ Department of Rehabilitation Medicine, Maoming people's Hospital, 525000 Maoming, China. ${ }^{3}$ Department of Rehabilitation Medicine, The Third Affiliated Hospital of Sun Yat-sen Univerity, 510630 Guangzhou, China.

References

1. Razaq, S., Azam Rathore, F.: An overview of pathophysiology, assessment and management strategies of post stroke shoulder subluxation. Pakistan Journal of Neurological Sciences (PJNS) 11(3), 42-48 (2016)

2. Domínguez-Carrillo, L.G., Arellano-Aguilar, J.G., Villasana-Delfín, O.A.: Shoulder pain and dysfunction in hemiplegia: effects of multiple application of functional electrical stimulation. Acta Médica Grupo Ángeles 3(4), 221-227 (2005)

3. Idowu, B.M., Ayoola, O.O., Adetiloye, V.A., Komolafe, M.A., Afolabi, B.I.: Sonographic detection of inferior subluxation in post-stroke hemiplegic shoulders. Journal of ultrasonography 17(69), 106 (2017)

4. Kumar, P., Mardon, M., Bradley, M., Gray, S., Swinkels, A.: Assessment of glenohumeral subluxation in poststroke hemiplegia: Comparison between ultrasound and fingerbreadth palpation methods. Physical therapy 94(11), 1622-1631 (2014)

5. Faghri, P.D., Rodgers, M.M., Glaser, R.M., Bors, J.G., Ho, C., Akuthota, P.: The effects of functional electrical stimulation on shoulder subluxation, arm function recovery, and shoulder pain in hemiplegic stroke patients. Archives of physical medicine and rehabilitation 75(1), 73-79 (1994)

6. Brooke, M.M., de Lateur, B.J., Diana-Rigby, G.C., Questad, K.A.: Shoulder subluxation in hemiplegia: effects of three different supports. Archives of physical medicine and rehabilitation 72(8), 582-586 (1991)

7. Södring, K.M.: Upper extremity orthoses for stroke patients. International journal of rehabilitation research. Internationale Zeitschrift fur Rehabilitationsforschung. Revue internationale de recherches de readaptation 3(1), 33-38 (1980) 
8. Zorowitz, R.D., Idank, D., Ikai, T., Hughes, M.B., Johnston, M.V.: Shoulder subluxation after stroke: a comparison of four supports. Archives of physical medicine and rehabilitation 76(8), 763-771 (1995)

9. Hartwig, M., Gelbrich, G., Griewing, B.: Functional orthosis in shoulder joint subluxation after ischaemic brain stroke to avoid post-hemiplegic shoulder-hand syndrome: A randomized clinical trial. Clinical rehabilitation 26(9), 807-816 (2012)

10. Boyd, E.A., Pepin, P., Szabo-Hartin, J.: Shoulder supports revisited: a canadian follow-up survey. Canadian Journal of Occupational Therapy 66(4), 161-168 (1999)

11. Stolzenberg, D., Siu, G., Cruz, E.: Current and future interventions for glenohumeral subluxation in hemiplegia secondary to stroke. Topics in stroke rehabilitation 19(5), 444-456 (2012)

12. Boyd, E.A., Goudreau, L., O'Rianin, M.D., Grinnell, D.M., Torrance, G.M., Gaylard, A.: A radiological measure of shoulder subluxation in hemiplegia: its reliability and validity. Archives of physical medicine and rehabilitation 74(2), 188-193 (1993)

13. Hall, J., Dudgeon, B., Guthrie, M.: Validity of clinical measures of shoulder subluxation in adults with poststroke hemiplegia. American Journal of Occupational Therapy 49(6), 526-533 (1995)

14. Hayes, K.W., Sullivan, J.E.: Reliability of a new device used to measure shoulder subluxation. Physical therapy 69(9), 762-767 (1989)

15. Boyd, E.A., Torrance, G.M.: Clinical measures of shoulder subluxation: their reliability. Canadian journal of public health = Revue canadienne de sante publique 83, 24-8 (1992)

16. de Gonzalez, A.B., Darby, S.: Risk of cancer from diagnostic x-rays: estimates for the uk and 14 other countries. The lancet 363(9406), 345-351 (2004)

17. Park, G.-Y., Kim, J.-M., Sohn, S.-I., Shin, I.-H., Lee, M.Y.: Ultrasonographic measurement of shoulder subluxation in patients with post-stroke hemiplegia. Journal of rehabilitation medicine 39(7), 526-530 (2007)

18. Allen, G., Wilson, D.: Ultrasound of the shoulder. European journal of ultrasound 14(1), 3-9 (2001)

19. Orengo, G., Lagati, A., Saggio, G.: Modeling wearable bend sensor behavior for human motion capture. IEEE Sensors Journal 14(7), 2307-2316 (2014)

20. Saggio, G., Sbernini, L.: New scenarios in human trunk posture measurements for clinical applications. In: 2011 IEEE International Symposium on Medical Measurements and Applications, pp. 13-17 (2011). IEEE

21. Adnan, N.H., Wan, K., Shahriman, A., Za'ba, S.K., Desa, H., Aziz, M.: The development of a low cost data glove by using flexible bend sensor for resistive interfaces. In: The 2nd International Malaysia-Ireland Joint Symposium on Engineering, Science and Business, pp. 579-587 (2012)

22. Saggio, G., Quitadamo, L.R., Albero, L.: Development and evaluation of a novel low-cost sensor-based knee flexion angle measurement system. The Knee 21(5), 896-901 (2014)

23. Saggio, G.: A novel array of flex sensors for a goniometric glove. Sensors and Actuators A: Physical 205, 119-125 (2014)

24. Dipietro, L., Sabatini, A.M., Dario, P.: A survey of glove-based systems and their applications. leee transactions on systems, man, and cybernetics, part c (applications and reviews) 38(4), 461-482 (2008)

25. Simone, L.K., Sundarrajan, N., Luo, X., Jia, Y., Kamper, D.G.: A low cost instrumented glove for extended monitoring and functional hand assessment. Journal of neuroscience methods 160(2), 335-348 (2007)

26. Saggio, G., Orengo, G.: Flex sensor characterization against shape and curvature changes. Sensors and Actuators A: Physical 273, 221-231 (2018)

27. Saggio, G.: Mechanical model of flex sensors used to sense finger movements. Sensors and Actuators A: Physical 185, 53-58 (2012)

28. Saggio, G., Pallotti, A., Sbernini, L., Errico, V., Di Paolo, F.: Feasibility of commercial resistive flex sensors for hand tracking applications. Sensors \& Transducers 201(6), 17 (2016)

29. Miller, L.J.: Developing Norm-referenced Standardized Tests. Psychology Press, ??? (1989)

30. Paci, M., Nannetti, L., Taiti, P., Baccini, M., Pasquini, J., Rinaldi, L.: Shoulder subluxation after stroke: relationships with pain and motor recovery. Physiotherapy Research International 12(2), 95-104 (2007)

31. Saggio, G., Riillo, F., Sbernini, L., Quitadamo, L.R.: Resistive flex sensors: a survey. Smart Materials and Structures 25(1), 013001 (2015)

32. Simone, L.K., Kamper, D.G.: Design considerations for a wearable monitor to measure finger posture. Journal of neuroengineering and rehabilitation 2(1), 1-10 (2005) 
Figures

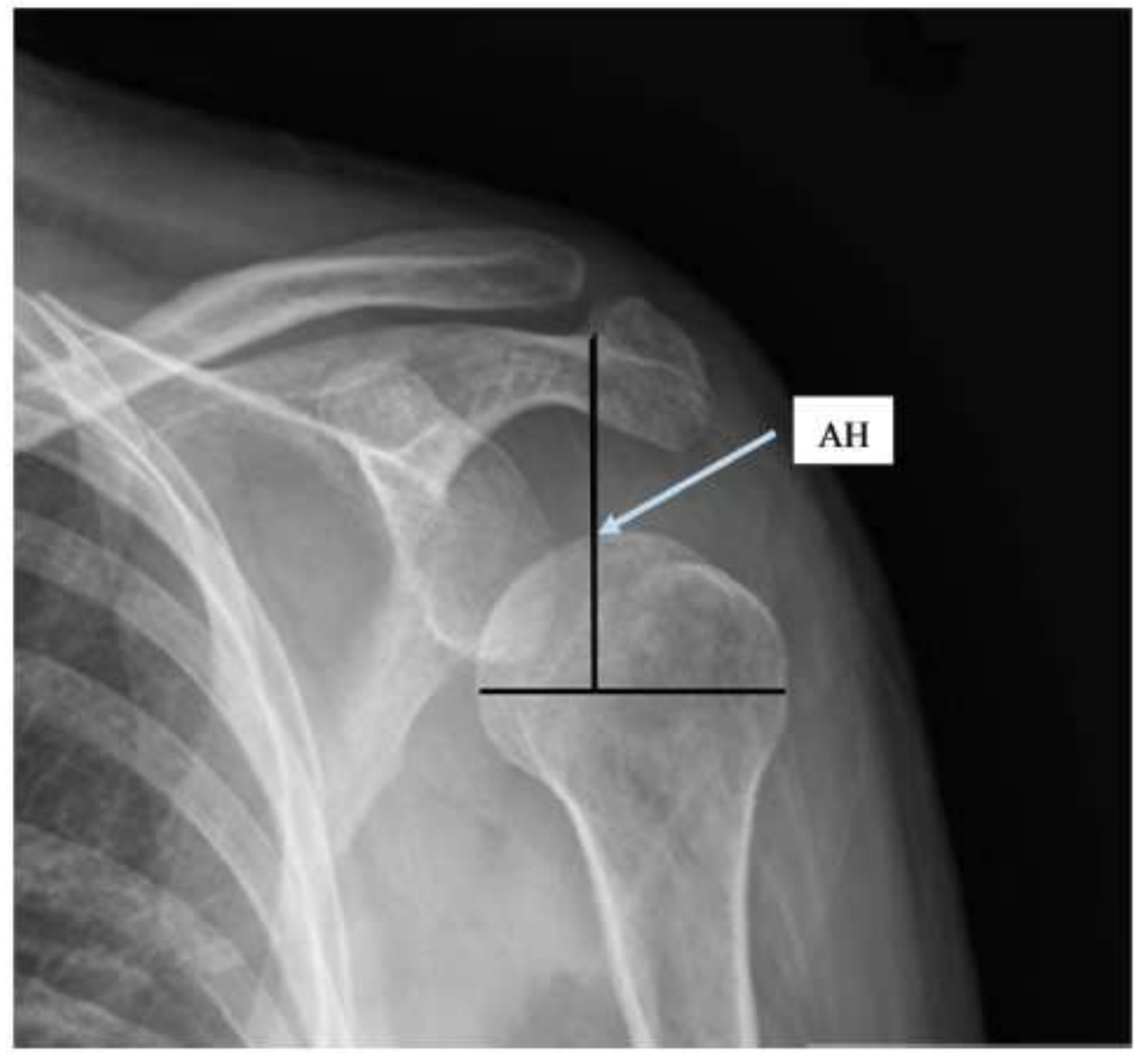

\section{Figure 1}

Measurement of GHS degree from radiograph, which measured from the acromial point to the central point of the humeral head.

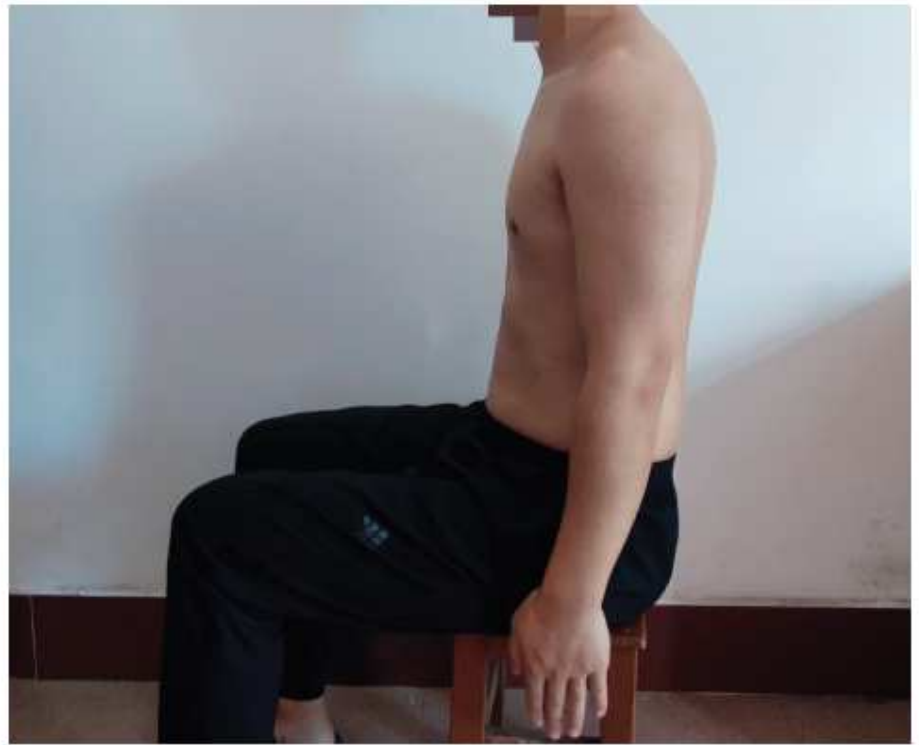

(a)

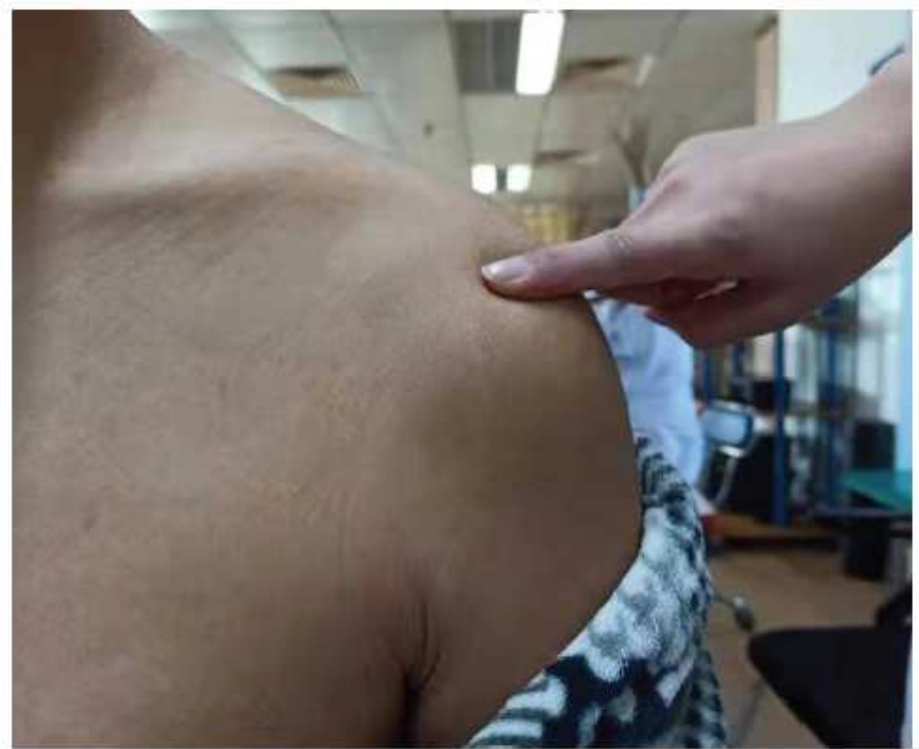

(b) 
Figure 2

Standardized position for fingerbreadth palpation data collection (a) and fingerbreadth palpation (b).

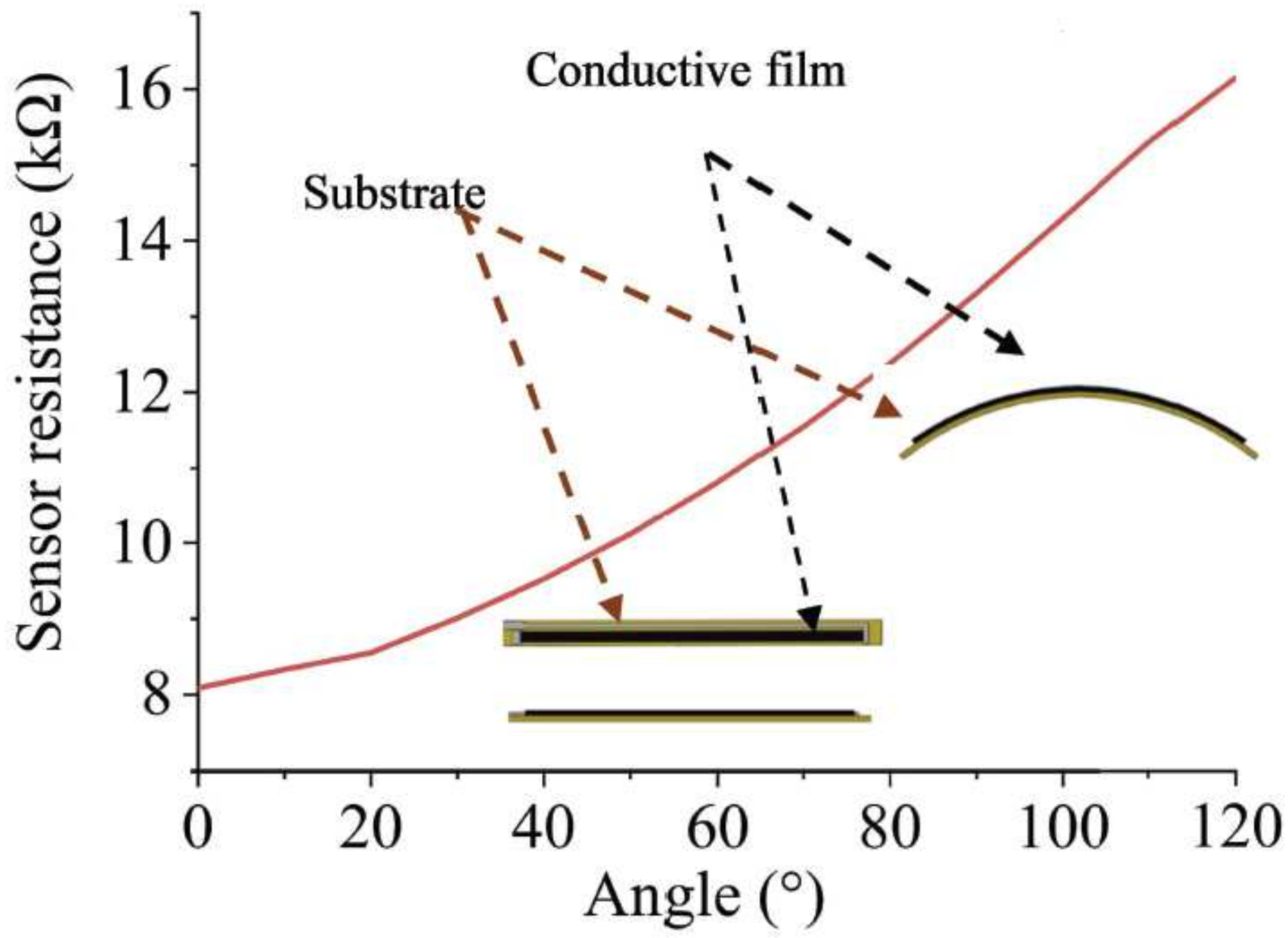

Figure 3

Scheme of the flex sensor. 


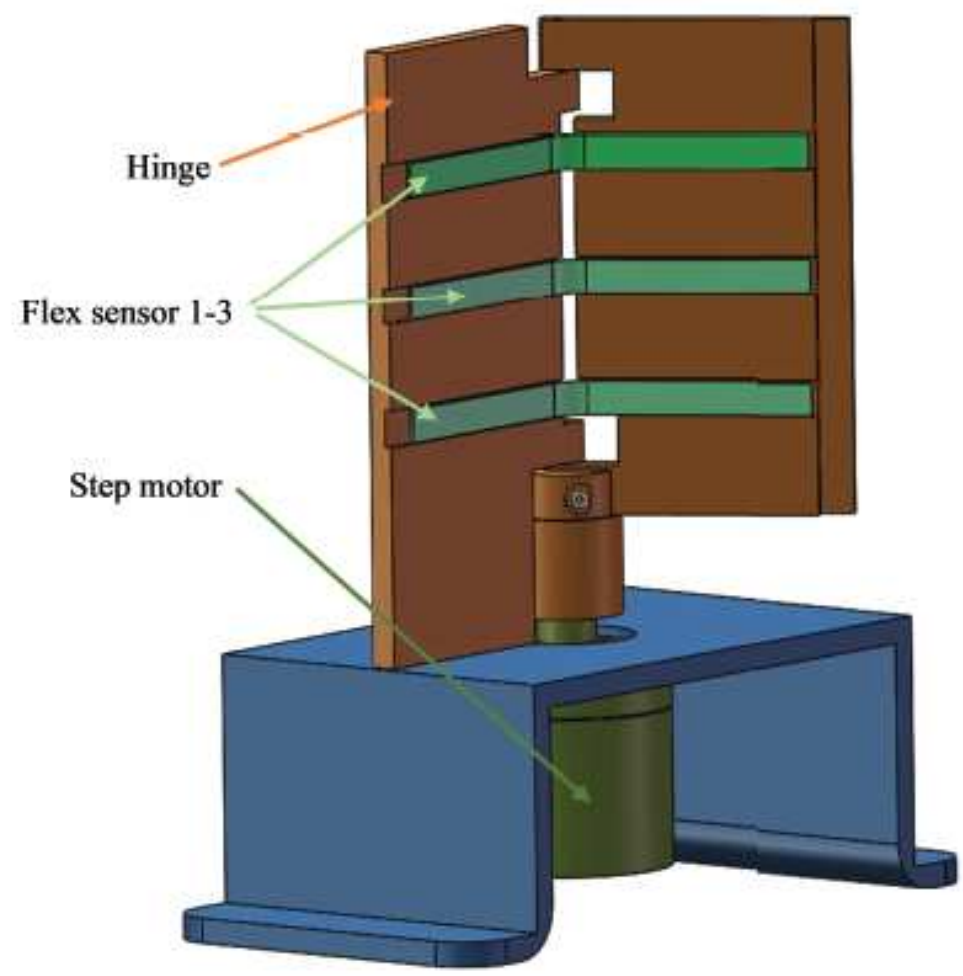

(a)

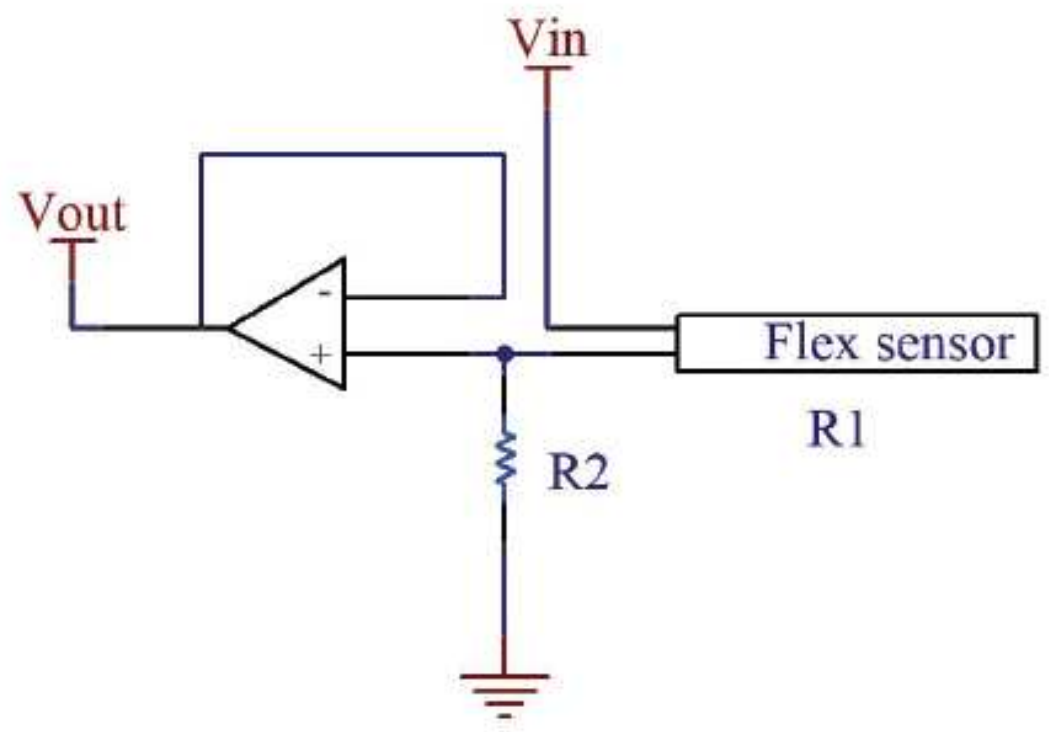

(b)

Figure 4

Experimental set-up used to characterize the sensor device against the bending angle (a) and basic circuit diagram of a flex sensor (b). 


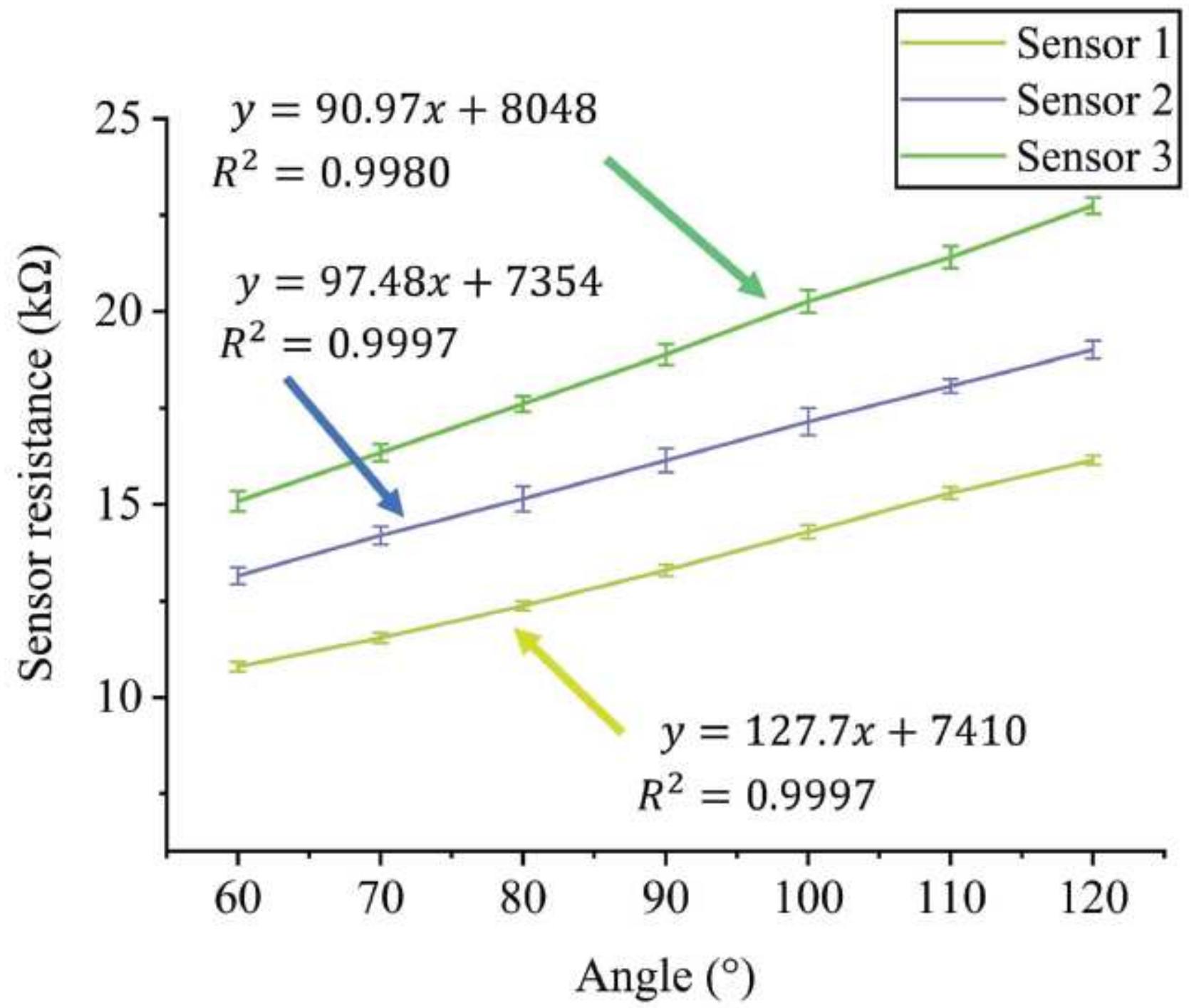

Figure 5

Static characterization and relationship of sensor resistance against outward bending angles, as total mean and standard deviation either inside measurements on the same sample and between 5 samples of each sensor. 


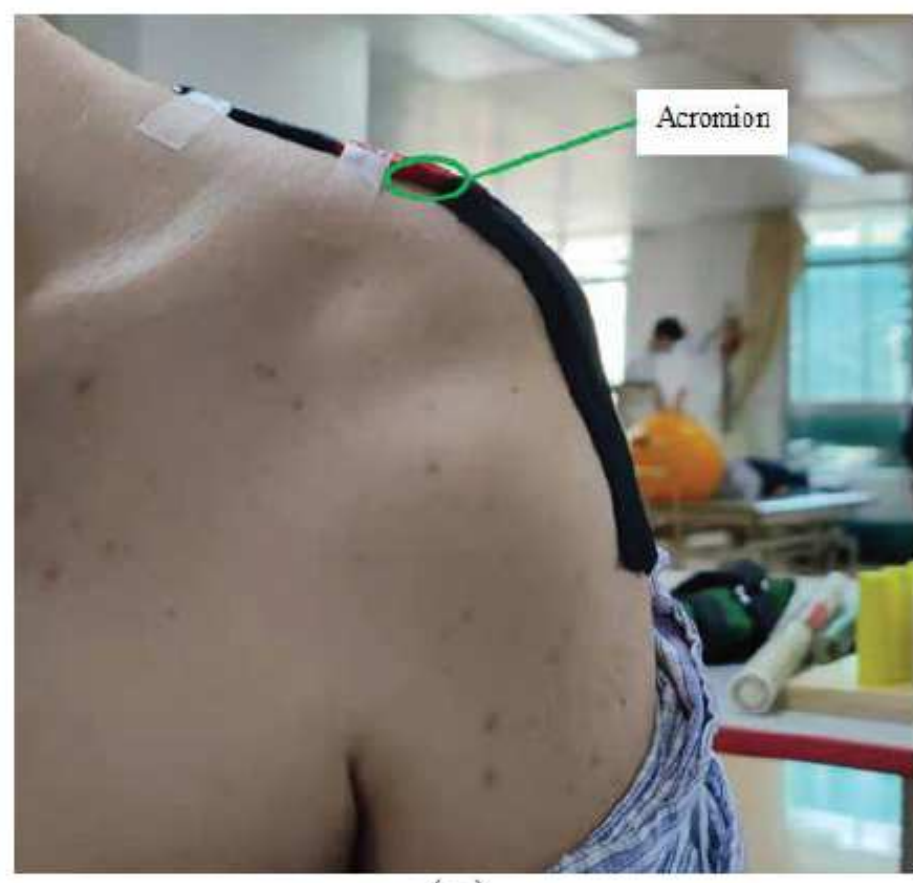

(a)

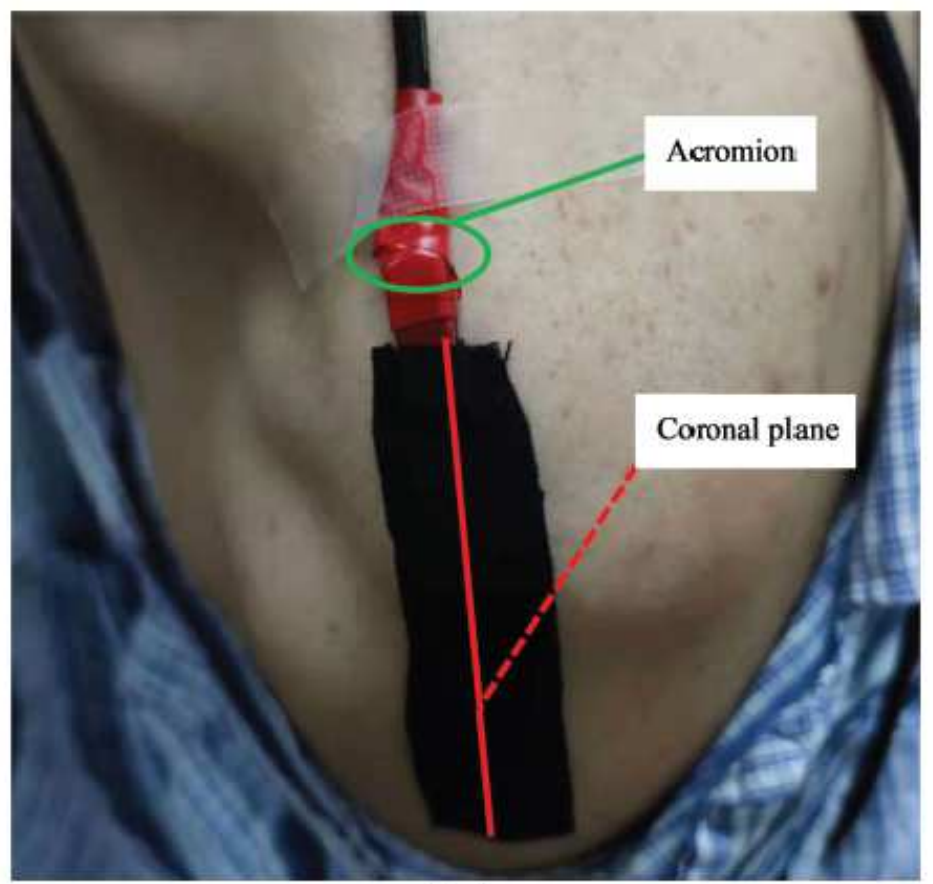

(b)

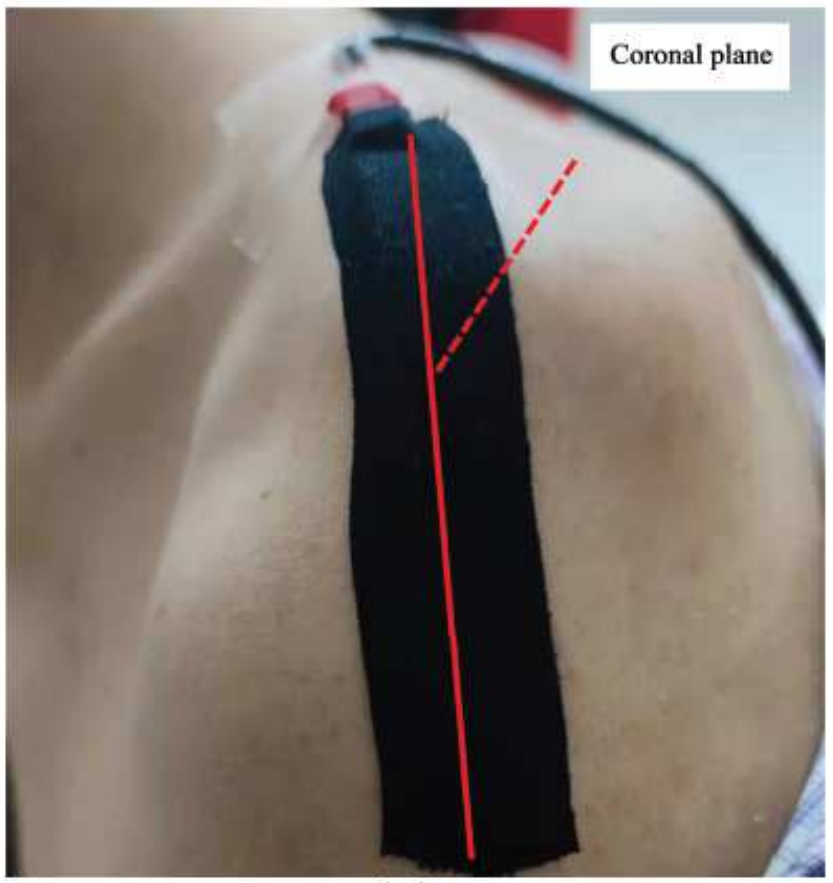

(c)

Figure 6

Front (a), left (b), and upper (c) views of GSB placed on the shoulder joint. 


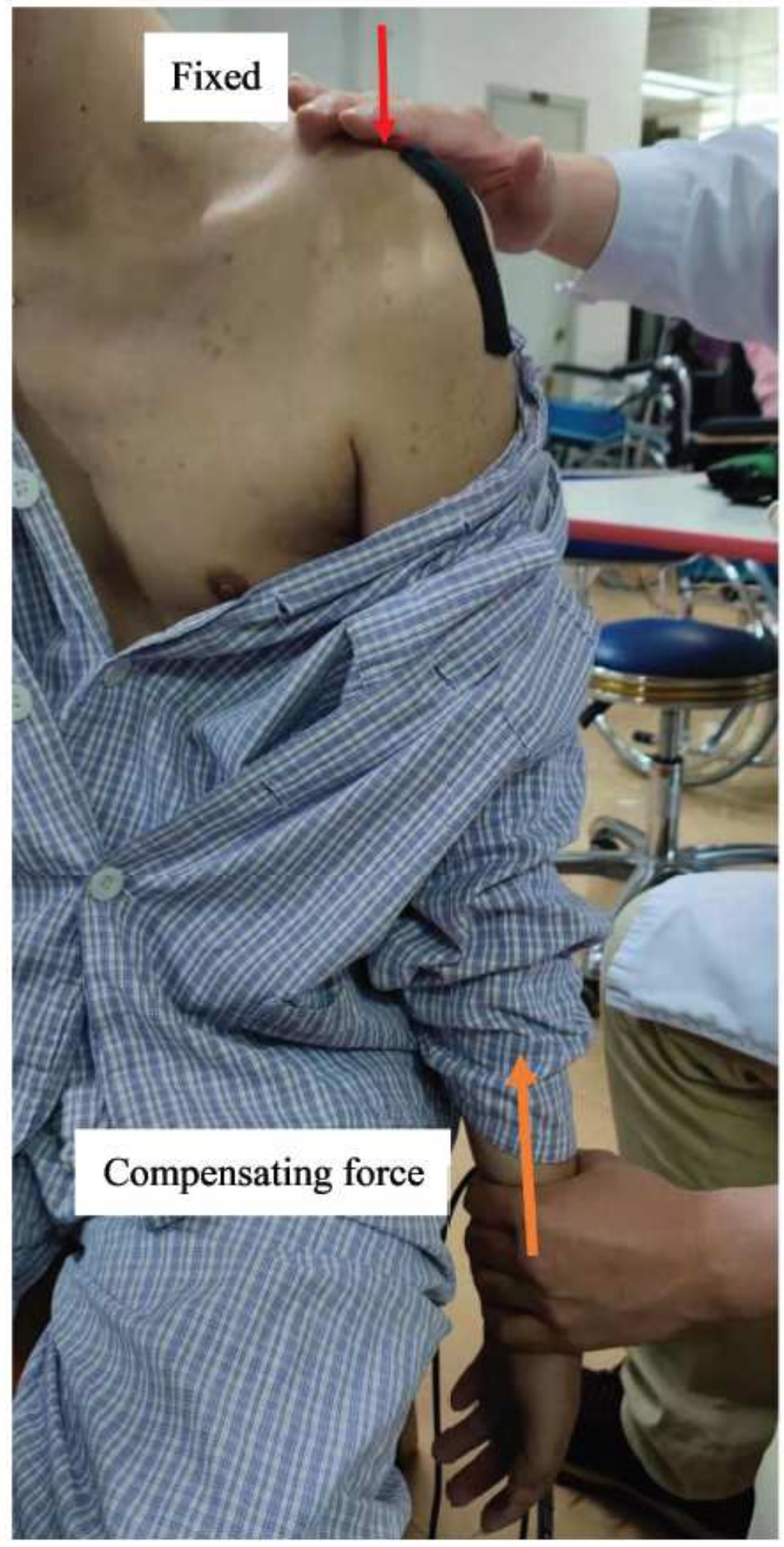

Figure 7

Therapist's action of reducing the patient's shoulder joint. 


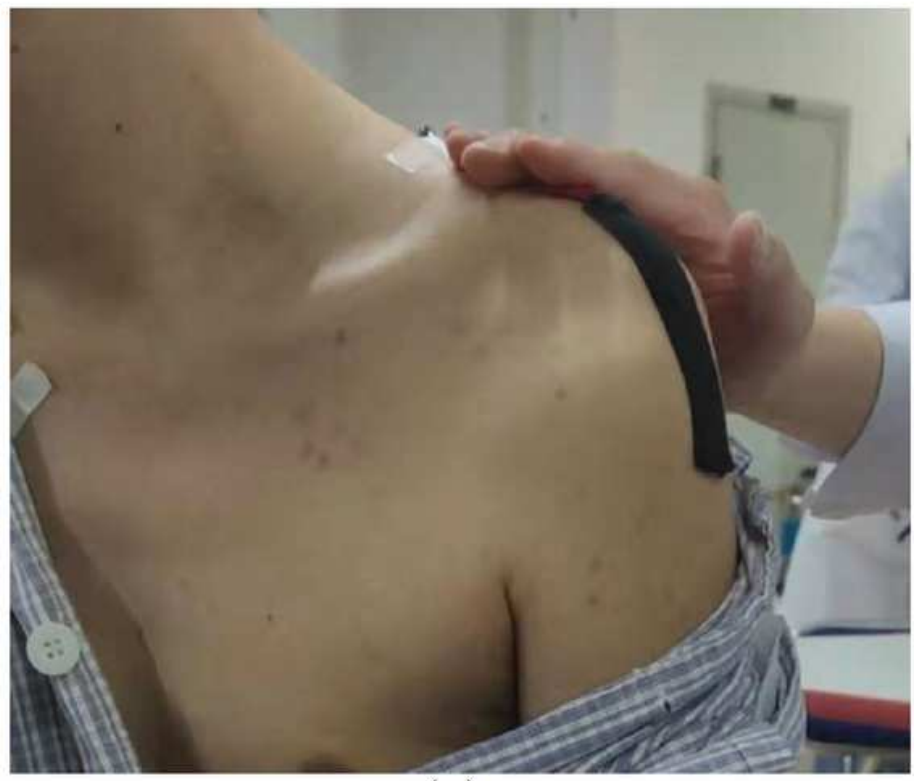

(a)

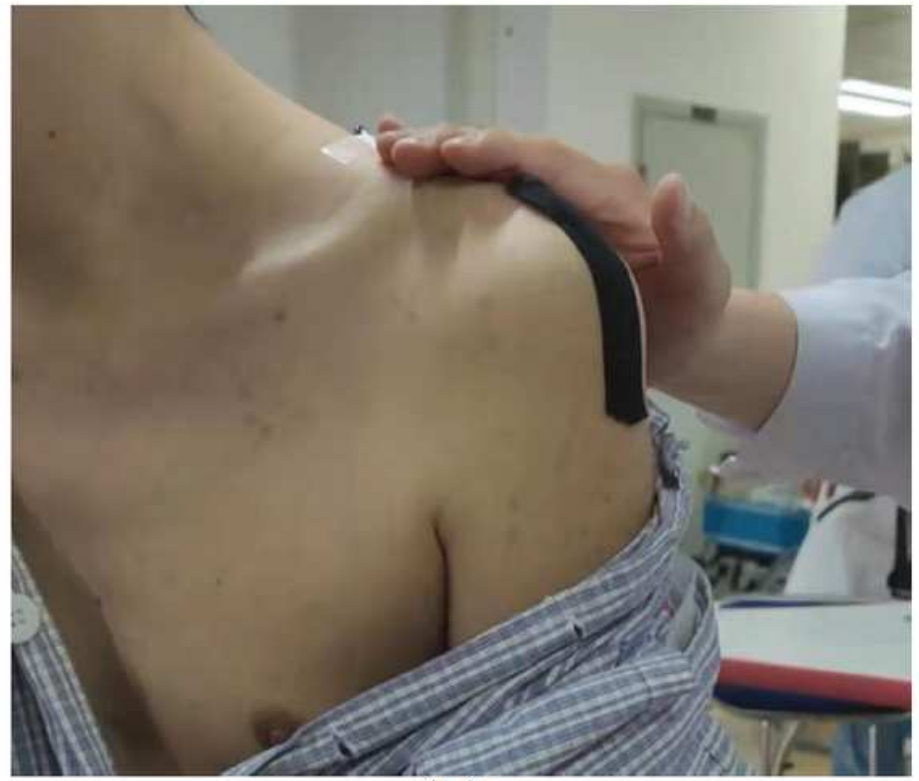

(b)

\section{Figure 8}

(a) The shape of shoulder the joint in subluxation. (b) The shape of the shoulder joint in reduction. 


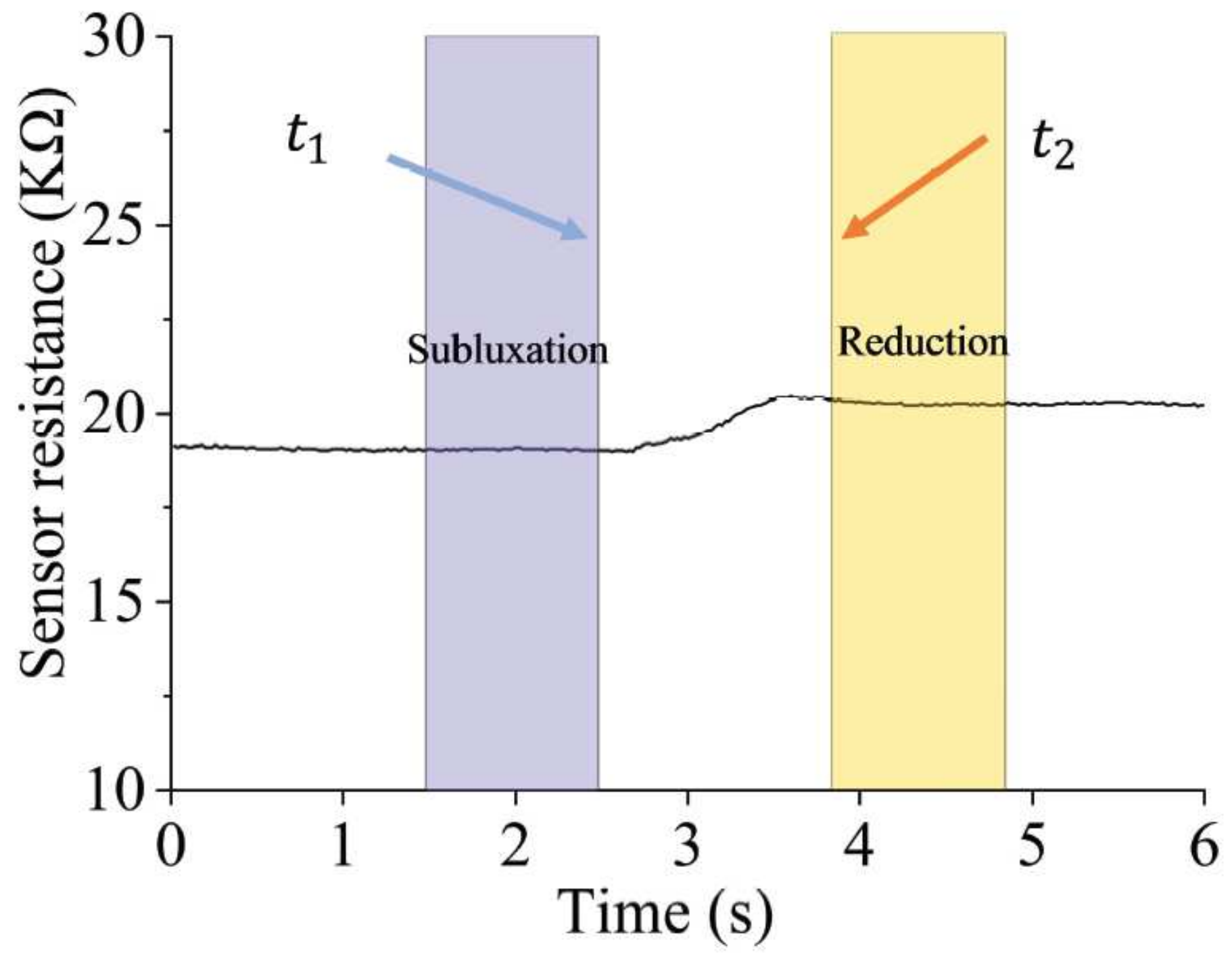

Figure 9

Example of data segmentation. 


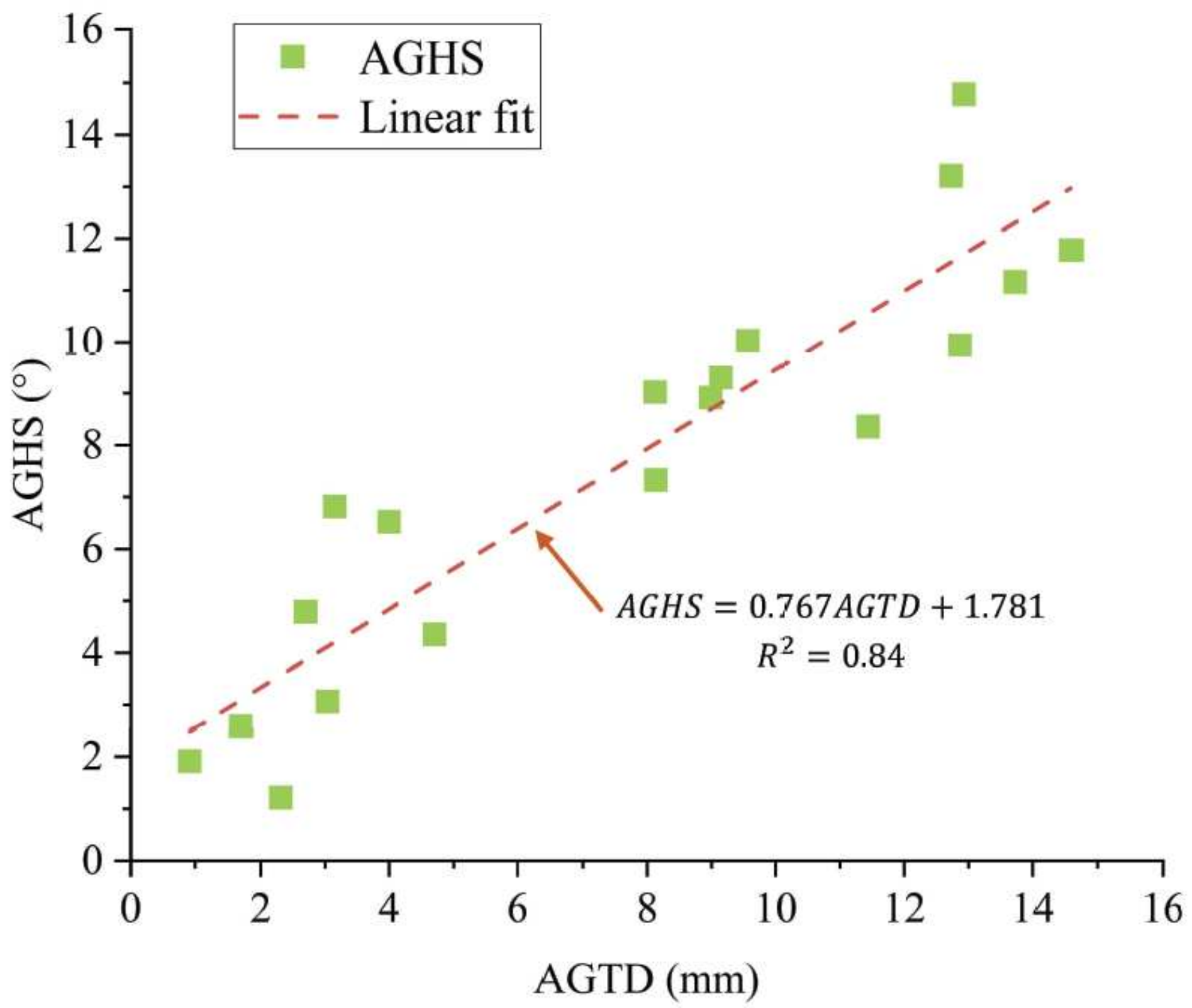

Figure 10

The relationship between the GHS degree measured by radiographic assessment (AGTD) and the GHS degree measured by Flex sensor (AGHS). 


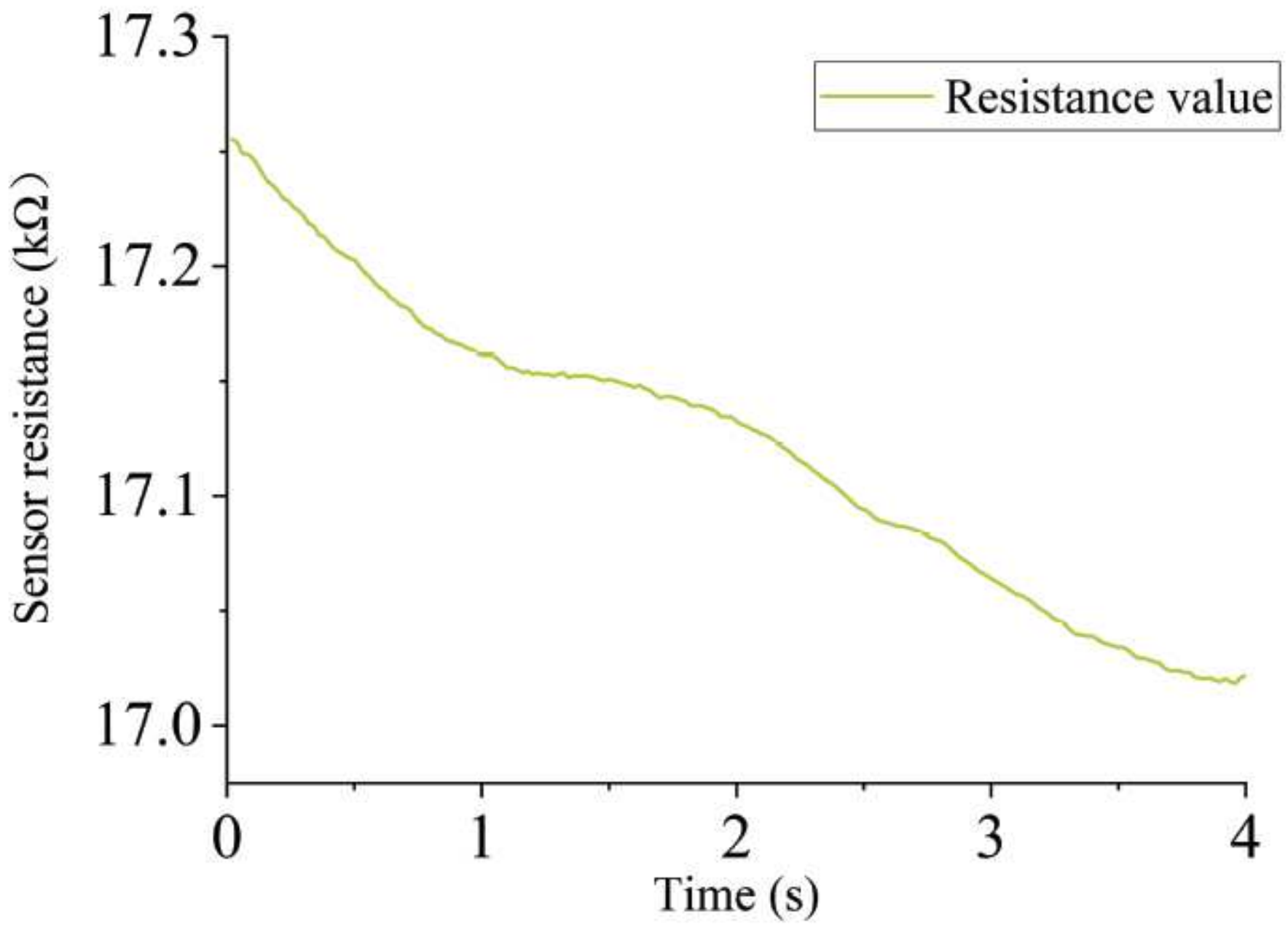

Figure 11

The drift of flex sensor resistance value with time.

\section{Supplementary Files}

This is a list of supplementary files associated with this preprint. Click to download.

- Therapistsactionofreducingthepatientsshoulderjoint..mp4 